GROSSE STIMMEN 
JENS MALTE FISCHER

\section{GROSSE STIMMEN}

Von Enrico Caruso bis Jessye Norman

VERLAG J. B. METZLER

STUTTGART · WEIMAR 
Die Deutsche Bibliothek - CIP-Einheitsaufnahme

\section{Fischer, Jens Malte:}

Grosse Stimmen : von Enrico Caruso bis Jessye Norman / Jens Malte Fischer. - Stuttgart : Metzler, 1993

ISBN 978-3-476-00893-0

ISBN 978-3-476-00893-0

ISBN 978-3-476-03461-8 (eBook)

DOI 10.1007/978-3-476-03461-8

Dieses Werk einschließlich aller seiner Teile ist urheberrechtlich geschützt. Jede Verwertung außerhalb der engen Grenzen des Urheberrechtsgesetzes ist ohne Zustimmung des Verlages unzulässig und strafbar. Das gilt insbesondere für Vervielfältigungen, Übersetzungen, Mikroverfilmungen und die Einspeicherung und Verarbeitung in elektronischen Systemen.

(C) 1993 Springer-Verlag GmbH Deutschland

Ursprünglich erschienen bei J.B. Metzlersche Verlagsbuchhandlung und Carl Ernst Poeschel Verlag GmbH in Stuttgart 1993 


\section{Inhalt}

\section{Sängerinnen und Sänger der Jahrhundertwende}

Enrico Caruso 1 - Fjodor Schaljapin 17 - Mattia Battistini 27 - Titta Ruffo 31 - Nellie Melba 39

Pol Plançon 44 - Marcel Journet 47 . Pasquale Amato 48 . Giuseppe De Luca 49 . Joseph Schwarz 51 - Leo Slezak 52 - John McCormack 55 - Ernestine SchumannHeink $57 \cdot$ Lilli Lehmann $60 \cdot$ Emmy Destinn 62 - Marcella Sembrich $63 \cdot$ Luisa Tetrazzini $65 \cdot$ Selma Kurz $67 \cdot$ Frieda Hempel 68 - Adelina Patti 70

\section{Erstes Intermezzo: Der Höhepunkt des italienischen dramatischen Gesangs - Otello, eine Rolle und ihre Sänger 73}

Einzelaufnahmen unter anderem von

Francesco Tamagno - Enrico Caruso · Giovanni Zenatello · John O'Sullivan · Renato Zanelli · Lauritz Melchior · Francesco Merli · Todor Masaroff · Wladimir Atlantow

Gesamtaufnahmen unter anderem von

Giovanni Martinelli · Ramón Vinay · Mario Del Monaco · Jon Vickers · James McCracken · Carlo Cossutta $\cdot$ Plácido Domingo $\cdot$ Luciano Pavarotti

\section{Sängerinnen und Sänger der zwanziger und dreißiger Jahre} 115

Alexander Kipnis 115 - Ezio Pinza $122 \cdot$ Richard Tauber $125 \cdot$ Tito Schipa 136 - Beniamino Gigli 143 - Aureliano Pertile 147 - Lauritz Melchior 155 - Rosa Ponselle 163 - Lotte Lehmann $169 \cdot$ Frida Leider 179 
Nazzareno De Angelis 188 - Tancredi Pasero $189 \cdot$ Michael Bohnen $190 \cdot$ Heinrich Schlusnus 191 - Gerhard Hüsch 193 - Willi Domgraf-Fassbaender 195 - Karl SchmittWalter 195 - Riccardo Stracciari 195 - Lawrence Tibbett 197 · Helge Rosvænge 199 - Giacomo Lauri-Volpi 201 · Joseph Schmidt 203 • Alfred Piccaver 206 · Giovanni Martinelli 209 · Sigrid Onegin 211 • Maria Jeritza 213 • Elisabeth Rethberg 215 . Meta Seinemeyer 217 • Tiana Lemnitz 220 • Ninon Vallin 221 • Claudia Muzio 222 . Maria Ivogün $224 \cdot$ Amelita Galli-Curci 226

\section{Zweites Intermezzo: Sprachgesang oder Belcanto? Wagners Sänger, die Bayreuther Schule und die Entwicklung des Wagner-Gesangs}

229

Emanuel List 236 - Ivar Andrésen 237 - Ludwig Weber 238 - Ludwig Hofmann 239 . Gottlob Frick 239 . Friedrich Schorr 241 . Rudolf Bockelmann 242 . Hans Hotter 244. Hans Hermann Nissen 246 . Ferdinand Frantz 247 . Margarete Klose 250 - Karin Branzell 250 • Maria Müller 252 . Germaine Lubin 254 · Helen Traubel 254 · Johanna Gadski 256 • Olive Fremstad 256 . Gertrude Kappel 257 . Florence Austral 257 • Birgit Nilsson 258 . Astrid Varnay 261 - Martha Mödl 263 - Kirsten Flagstad 264 - Catarina Ligendza 266 • Hildegard Behrens 267 - Sándor Kónya 271 · Jess Thomas 272 . Franz Völker 272 · Jussi Björling 273 · Heinrich Knote 274 • Max Lorenz 275 - Wolfgang Windgassen 279 · Richard Schubert 281 . Set Svanholm 281 . Alberto Remedios 282 - Helge Brilioth 282 - Hans Hopf 283 - Ludwig Suthaus 284 - Jacques Urlus 285 • James King 286 - Peter Hofmann 286 - René Kollo 287 · Siegfried Jerusalem 288 - Reiner Goldberg 289

\section{Sängerinnen und Sänger der vierziger und fünfziger Jahre 293}

Boris Christoff 293 • George London 296 • Jussi Björling 301 • Ramón Vinay 309 - Kathleen Ferrier 315 . Elisabeth Schwarzkopf 321 - Maria Callas 325

Cesare Siepi 333 · Josef Greindl 334 - Paul Schöffler 335 - Tito Gobbi 337 - Leonard Warren 338 - Robert Merrill 340 - Josef Metternich 341 - Ferruccio Tagliavini 342 . Giuseppe Di Stefano 344 · Léopold Simoneau 345 - Anton Dermota 347 · Peter Anders 349 - Rudolf Schock 350 - Peter Pears 351 - Richard Tucker 353 - Mario Del Monaco 355 - Giulietta Simionato 357 - Zinka Milanov 359 - Renata Tebaldi 361 . Victoria de los Angeles 363 - Ljuba Welitsch 364 - Sena Jurinac 366 - Maria Cebotari 367 . Elisabeth Grümmer 369 · Irmgard Seefried 371 • Erna Berger 373 - Maria Stader 374 
Inhalt

\section{Drittes Intermezzo: Die Merkwürdigen und die Außenseiter, die Verkannten und die Unbekannten}

377

Deutschsprachige Sängerinnen und Sänger 381 - Italienische und spanische Sängerinnen und Sänger 388 - Französischsprachige Sängerinnen und Sänger 393 - Anglo-amerikanische Sängerinnen und Sänger 397 · Skandinavische Sänger 402 - Osteuropäische Sängerinnen und Sänger 404

\section{Sängerinnen und Sänger der sechziger und siebziger Jahre} 411

Nicolai Ghiaurov 411 - Dietrich Fischer-Dieskau 415 - Carlo Bergonzi 425 . Fritz Wunderlich 428 - Nicolai Gedda 436 . Christa Ludwig $443 \cdot$ Janet Baker $447 \cdot$ Marilyn Horne $450 \cdot$ Joan Sutherland $452 \cdot$ Mirella Freni 458

Martti Talvela 460 - Karl Ridderbusch 460 - Franz Crass 461 - Ettore Bastianini 462 - Piero Cappuccilli 462 · Gérard Souzay 463 - Sherrill Milnes 464 - Walter Berry 465 - Thomas Stewart 466 . Hermann Prey 467 . Jon Vickers 469 . Franco Corelli 471 . Alfredo Kraus 473 - Peter Schreier 475 - Stuart Burrows 477 - Fiorenza Cossotto 477 - Brigitte Fassbaender 479 . Teresa Berganza 481 . Frederica Von Stade 482 . Gwyneth Jones 483 - Leonie Rysanek 485 - Régine Crespin 486 . Gundula Janowitz 488 . Elisabeth Söderström 489 - Lisa della Casa 491 . Ileana Cotrubas 492 . Teresa Stratas 494 . Leontyne Price 496 . Shirley Verrett 497 . Grace Bumbry 498 - Martina Arroyo 499 - Renata Scotto 499 . Montserrat Caballé 501 - Beverly Sills 503 - Anja Silja $504 \cdot$ Helen Donath $505 \cdot$ Anneliese Rothenberger $506 \cdot$ Erika Köth 507 - Rita Streich 507 - Edith Mathis $508 \cdot$ Reri Grist 509

\section{Viertes Intermezzo: Jüdische Sänger - Eigenart und Schicksal}

511

\section{Sängerinnen und Sänger der Gegenwart}

523

Plácido Domingo 523 - Luciano Pavarotti $529 \cdot$ José Carreras $534 \cdot$ Agnes Baltsa $540 \cdot$ Julia Varady $543 \cdot$ Lucia Popp $545 \cdot$ Edita Gruberová $548 \cdot$ Jessye Norman 551

Paata Burchuladse 557 Jewgeni Nesterenko 558 - Matti Salminen 559 J Jako Ryhänen 559 - Kurt Moll 559 - Hans Sotin 560 - Matthias Hölle 560 - Samuel Ramey 561 - Ruggero Raimondi 563 · José Van Dam 564 - Ferruccio Furlanetto 565 - Siegmund Nimsgern 566 - Simon Estes $567 \cdot$ James Morris 568 - Robert Hale 569 · John Tom- 
linson 570 - Bernd Weikl 570 - Andreas Schmidt 572 - Dmitri Chworostowski 573 . Thomas Allen 574 - Alan Titus 574 · Thomas Hampson 575 • Leo Nucci 576 • Giorgio Zancanaro 577 . Renato Bruson 577 . Hans Peter Blochwitz 579 . Deon van der Walt 579 · Kurt Streit 579 · Jerry Hadley 579 • Gösta Winbergh 579 · Francisco Araiza 580 - Chris Merritt 580 - Frank Lopardo $581 \cdot$ Rockwell Blake $581 \cdot$ Ramón Vargas 581 - Luis Lima 581 - Neil Shicoff 582 . Franco Bonisolli 583 - Wladimir Atlantow 584 - Jochen Kowalski 585 - Robert Tear $585 \cdot$ Kenneth Riegel 586 - Philip Langridge 586 - Lucia Valentini Terrani 587 - Cecilia Bartoli 587 - Ann Murray 587 . Hanna Schwarz 588 - Waltraud Meier 588 - Marjana Lipovšek 589 . Anne-Sofie von Otter 590 - Eva Marton 590 . Cheryl Studer 591 - Margaret Price 592 - Kiri te Kanawa 593 - Katia Ricciarelli 594 - Mara Zampieri 595 - Lucia Aliberti 595 - Gabriela Beňačková 596 - Kathleen Battle 596 • Barbara Hendricks 596 - Catherine Malfitano 597

\section{Glossar}

599

\section{Literaturverzeichnis}

617

\section{Personenregister}




\section{Vorwort}

Das Buch stellt den Versuch dar, in einem Band ein knappes Jahrhundert in der Geschichte des Kunstgesangs zu überblicken. Es beginnt zu dem Zeitpunkt, an dem die Geschichte der Schallaufzeichnung es ermöglicht, Gesangsleistungen mit einer ausreichenden Wiedergabetreue aufzuzeichnen, also um die Jahrhundertwende, und hier mit der epochalen Erscheinung Enrico Carusos. Eine auch nur annähernde Vollständigkeit, wie sie mehrbändige Werke lexikalischer Natur geben können, war nicht angestrebt. Angesichts der räumlichen $\mathrm{Be}-$ schränkung mußte eine Grundsatzentscheidung fallen: Werden möglichst viele Namen genannt, oder wird auf Ausführlichkeit im Einzelfall beharrt? Der Autor hat sich für die zweite Lösung entschieden, wohl wissend, daß dies schmerzliche Verluste nach sich zieht. Es liegt ihm daran, seinen Lesern zu versichern, da $\beta$ er eine tränendurchfeuchtete Liste in seinem Schreibtisch verwahrt mit den Namen jener Sängerinnen und Sänger, die er nicht aufnehmen konnte - jede einzelne Entscheidung gegen einen Namen ist ihm schwergefallen, zumal die Qualitätskriterien, die solchen Entscheidungen zugrunde lagen, keineswegs immer eindeutig zu bestimmen waren. Da die angestrebte Ausfuhhrlichkeit dennoch nicht allen behandelten Künstlern gleichermaßen gegönnt werden konnte, nimmt das Buch eine Zweiteilung vor: Jene Künstler, deren höchster Rang international unbestritten ist, erhielten ein größeres Porträt zugeordnet, die anderen ein kleineres. Dies bedeutet keineswegs durchgehend eine Einteilung in "erstklassig" und "zweitklassig"; manchmal ist ein Sänger nur in der ersten Kategorie eingeordnet, weil er zeittypischer, repräsentativer ist, nicht immer ist er deshalb technisch, musikalisch, interpretatorisch besser als ein anderer, der sich in der zweiten Kategorie befindet.

Das Buch bemüht sich vor allem um eines (und unterscheidet sich darin von vergleichbaren Publikationen): Nicht nur die gesangliche Leistung soll anhand der Schallplatten einer sezierenden Beurteilung unterzogen werden, son- 
dern vor allem soll hinter den Stimmen und Aufnahmen die Signatur des singenden Menschen deutlich werden; das Biographische, auch Anekdotische, wird daher nicht verschmäht. Was die gesangstechnische Beurteilung angeht, so weiß der Autor sich einig mit den Fachleuten für die Gesangskunst, daß sie in einem viel höheren $\mathrm{Ma} \beta$ objektivierbar ist, als weithin angenommen wird. Er versucht dies in einem gesangstechnischen Glossar darzulegen, das die wichtigsten Begriffe erklärt. Um die chronologische Folge der Porträts aufzulockern, aber auch, um eine Vertiefung des Blickes zu erreichen, sind zwischen die Porträtkapitel vier Exkurse, Intermezzi genannt, eingeschoben, die zum Teil querschnittartig vorgehen (das Wagner-Intermezzo), oder an einem speziellen Punkt (Verdis Otello) gewissermaßen eine Tiefenbohrung vornehmen. Wagner und Verdi werden in ihrer Einwirkung auf die Geschichte der Gesangskunst also bevorzugt behandelt. Vergleichbare Exkurse wären natürlich auch denkbar gewesen für den Mozart-Gesang, die italienische Belcanto-Oper, für Puccini oder Strauss. Hier mußte eine Auswahl getroffen werden, bei der persönliche Interessenschwerpunkte des Autors den Ausschlag gegeben haben.

Eine gewisse Mühe macht sich das Buch mit den "Hinweisen«. Die Leser werden es vielleicht begrüßen, bei den größeren Porträts die wichtigste Literatur über die betreffenden Sängerpersönlichkeiten genannt zu bekommen (auch wenn diese oft nicht in deutscher Sprache erschienen ist) und außerdem wichtige Recitals auf CD. Operngesamtaufnahmen mit den Sängern können nur gelegentlich aufgefuihrt werden, als Ausgleich sind im Anschluß an das Vorwort zentrale Opernaufnahmen mit historischem Charakter genannt. Bei den kleineren Porträts werden meist nur die Labels genannt, bei denen sich Recitals der Sänger finden lassen (im abschließenden Gegenwartskapitel wurde auch darauf verzichtet). Als die Arbeit an dem Buch begonnen wurde, enthielten die "Hinweise" noch reichhaltige Angaben zu Langspielplatten. Die in ihrem Tempo nicht vorhersehbare, geradezu brutale Verdrängung der Langspielplatte durch die Compact Disc läßt solche Hinweise nicht mehr sinnvoll erscheinen. Ein gewisses Trostpflaster ist die ebenfalls nicht erwartete Fülle von historischen Opern- und Gesangsaufnahmen auf CD. Insgesamt gilt sicher für die Leser dieses Buches und ihre Suche nach einschlägigen Tonträgern Parsifals Wort zu Kundry: "Du weißt, wo du mich wiederfinden kannst!" (Die Angaben haben den Stand des Jahresendes 1992.)

Der Autor möchte seinen Dank ausdrücken: Renate Treiber und vor allem Raphaela Rupert haben sich um das Manuskript verdient gemacht. Tamara Barzantny hat mit Korrektur gelesen und sich die große Mühe des Registers aufgeladen. Kurt Malisch hat das Manuskript einer kritischen Durchsicht unterzogen. Sein unübertreffliches Detailwissen in allen Bereichen, die Sängerinnen und Sänger betreffen, hat das Buch vor manchen Irrtümern bewahrt. 
Uwe Steffen hat nicht nur mit großem Satz-, sondern auch Sachverstand das Manuskript in die endgültige Buchform gebracht. Uwe Schweikert schließlich hat das Buch in einer kritischen Situation mit offenen Armen aufgenommen.

\section{Hinweise}

Eine Vorform des Wagner-Intermezzos erschien 1986 in der Zeitschrift Opernwelt. In Ergänzung des Literaturverzeichnisses sollen hier zunächst Handbücher genannt werden, in denen sich wichtige Informationen zur Sichtung des immensen tönenden Materials befinden, das auf LP und CD vorlag oder vorliegt. Da ist zunächst das unverzichtbare Standardwerk, das von Alan Blyth herausgegeben wurde: Opera on Record (3 Bände, London 1979, 1983, 1984). Einen historischen Katalog bietet Opern auf Schallplatte 1900-1962 (Wien 1974). Zur schnellen Orientierung sehr nützlich ist Karl Löbls und Robert Werbas Opern auf Schallplatte (2 Bände, Düsseldorf 1983). Für Spezialisten historischer Aufnahmen interessant ist Julian Morton Moses Collectors Guide to American Recordings 1895-1925, der zuerst New York 1949 erschien und dann in einem Reprint (New York 1977). Die besten Diskographien zu Sängern finden sich (soweit es keine Standardwerke zu ihnen gibt) in der englischen Spezialzeitschrift The Record Collector und der englischen Opernzeitschrift Opera. Ein nützliches Verzeichnis von Diskographien zur klassischen Musik und ihren Interpreten ist Michael H. Grays und Gerald D. Gibsons Bibliography of Discographies. Band 1: Classical Music, 1925-1975 (New York/London 1977).

Die speziellen CD-Hinweise bei den einzelnen Sängerinnen und Sängern sollen hier ergänzt werden durch Hinweise auf aktuelle $C D$-Anthologien mit wichtigen historischen Gesangsaufnahmen:

The Great Tenors, 2 CDs, Pearl

Tenors of the Bolschoi, 2 CDs, Pearl

Covent Garden on Record: A History, 12 CDs, Pearl

Divas, 2 CDs, Nimbus

Great Singers, 2 CDs, Nimbus

18 ténors d'expression française, $1 \mathrm{CD}$, Music Memoria

Les Introuvables du chant mozartien, 4 CDs, EMI

The Harold Wayne Collection (Raritäten), 7 CDs, Symposium

The Record of Singing Vol. 4 (1939-1955), 7 CDs, EMI

Berühmte italienische Baritone, $2 \mathrm{CDs}$, Memories

Famous Russian Singers, 6 CDs, Pearl

Abschließend eine Liste mit 25 historischen Opernaufnahmen, die der Autor seinen Lesern empfiehlt, einzig unter dem Gesichtspunkt der sängerischen Qualität. Vor allem 
bei den Live-Mitschnitten, die durch die Angabe des Opernhauses bezeichnet sind, müssen zum Teil erhebliche Abstriche an der technischen Qualität gemacht werden.

Vincenzo Bellini, Norma: Vittorio Gui; Maria Callas, Ebe Stignani, Mirto Picchi (Covent Garden London 1952); Legato.

Hector Berlioz, Les Troyens: Georges Prêtre; Nicolai Gedda, Marilyn Horne, Shirley Verrett (RAI Rom 1969); Melodram.

Georges Bizet, Carmen: Thomas Beecham; Victoria de los Angeles, Nicolai Gedda, Ernest Blanc (1958); EMI.

Luigi Cherubini, Medea: Leonard Bernstein; Maria Callas, Fedora Barbieri, Gino Penno (Scala Mailand 1953); Melodram.

Giacomo Meyerbeer, Le Prophète: Henry Lewis; Nicolai Gedda, Marilyn Horne (RAI Turin 1970); Myto.

Wolfgang Amadeus Mozart, Cosi fan tutte: Fritz Busch; Ina Souez, Luise Helletsgruber, Heddle Nash, Willi Domgraf-Fassbaender (Glyndebourne 1935); Pearl/EMI.

Wolfgang Amadeus Mozart, Don Giovanni: Bruno Walter; Ezio Pinza, Elisabeth Rethberg, Virgilio Lazzari (Salzburg 1937); Legato.

Wolfgang Amadeus Mozart, Le nozze di Figaro: Erich Kleiber; Cesare Siepi, Hilde Güden, Fernando Corena (1955); Decca.

Wolfgang Amadeus Mozart, Die Zauberflöte: Thomas Beecham; Wilhelm Strienz, Helge Rosvænge, Erna Berger, Tiana Lemnitz (1937/38); Pearl/EMI.

Giacomo Puccini, La Bohème: Thomas Beecham; Victoria de los Angeles, Jussi Björling, Robert Merrill (1956); EMI.

Giacomo Puccini, Tosca: Victor De Sabata; Maria Callas, Giuseppe Di Stefano, Tito Gobbi (1953); EMI.

Richard Strauss, Der Rosenkavalier. a) gekürzt: Robert Heger; Lotte Lehmann, Maria Olszewska, Elisabeth Schumann, Richard Mayr (1933); Pearl; b) Erich Kleiber; Maria Reining, Sena Jurinac, Hilde Güden, Ludwig Weber (1954); Decca.

Giuseppe Verdi, Aida: Carlo Sabajno; Dusolina Giannini, Aureliano Pertile, Irene Minghini-Cattaneo (1929); Pearl.

Giuseppe Verdi, Un ballo in maschera: Ettore Panizza; Jussi Björling, Alexander Svéd, Zinka Milanov, Bruna Castagna (Metropolitan Opera New York 1940); Myto.

Giuseppe Verdi, Don Carlos: Fritz Stiedry; Cesare Siepi, Jussi Björling, Robert Merrill, Fedora Barbieri (Metropolitan Opera New York 1950); Myto.

Giuseppe Verdi, La forza del destino: Dimitri Mitropoulos; Renata Tebaldi, Fedora Barbieri, Mario Del Monaco, Aldo Protti, Cesare Siepi (Florenz 1953); Foyer. 
Giuseppe Verdi, Otello: Fritz Busch; Ramón Vinay, Licia Albanese, Leonard Warren (Metropolitan Opera New York 1948); Melodram.

Giuseppe Verdi, Simon Boccanegra: Ettore Panizza; Elisabeth Rethberg, Lawrence Tibbett, Giovanni Martinelli, Ezio Pinza, Leonard Warren (Metropolitan Opera New York 1939); Melodram.

Giuseppe Verdi, La Traviata: Ettore Panizza; Rosa Ponselle, Lawrence Tibbett, Frederick Jagel (Metropolitan Opera New York 1935); Pearl.

Richard Wagner, Der fliegende Holländer: Antal Dorati; George London, Leonie Rysanek, Giorgio Tozzi (1960); Decca.

Richard Wagner, Die Meistersinger von Nürnberg: Artur Bodanzky; Friedrich Schorr, Elisabeth Rethberg, René Maison (Metropolitan Opera New York 1936); Music and Arts.

Richard Wagner, Tristan und Isolde: Wilhelm Furtwängler; Kirsten Flagstad, Ludwig Suthaus (1952); EMI.

Richard Wagner, Der Ring des Nibelungen: a) Wilhelm Furtwängler; Ferdinand Frantz, Martha Mödl, Ludwig Suthaus (RAI Rom 1953); EMI; b) Wilhelm Furtwängler; Ferdinand Frantz, Max Lorenz, Kirsten Flagstad, Set Svanholm (Scala Mailand 1950); Virtuoso.

Richard Wagner, Die Walküre: a) I. Akt: Bruno Walter; Lauritz Melchior, Lotte Lehmann, Emanuel List (1935); EMI; b) Erich Leinsdorf; Friedrich Schorr, Astrid Varnay, Helen Traubel, Lauritz Melchior, Alexander Kipnis (Metropolitan Opera New York 1941); Myto.

Richard Wagner, Siegfried: Artur Bodanzky; Lauritz Melchior, Kirsten Flagstad, Friedrich Schorr (Metropolitan Opera New York 1937); Music and Arts. 